\title{
Pacemaker Programming, Physiologic Pacing Settings, and Clinical Outcomes in Real-world Practice: Results from the OPTI-MIND Clinical Study
}

\author{
MAURO BIFFI, MD ${ }^{1}$, GEMMA PELARGONIO, MD ${ }^{2}$, ALEŠ HAVLÍČEK, MD ${ }^{3}$, PAOLO ROSSI, MD ${ }^{4}$, \\ DONATO MELISSANO, MD ${ }^{5}$, GERT KALTOFEN, MD ${ }^{6}$, MAURIZIO MENICHELLI, $\mathrm{MD}^{7}$, \\ GABRIELA KALISKA, MD ${ }^{8}$, RAFAEL ROMERO, MD ${ }^{9}$, CIRO GUASTAFERRO, MD ${ }^{10}$, \\ GIOVANNI RACITI, MD ${ }^{11}$, SCOTT WEHREMBERG, MD ${ }^{11}$, GIUSEPPE BORIANI, MD ${ }^{1}$ and \\ TIBOR MALACKY, MD ${ }^{12}$ \\ ${ }^{1}$ Institute of Cardiology, University of Bologna, Bologna, Italy \\ ${ }^{2}$ Cardiovascular Science Department, Catholic University of the Sacred Heart, Rome, Italy \\ ${ }^{3}$ Artur Koblitz s.r.o., Arrhythmia services, Pardubice, Kyiv Czech Republic \\ ${ }^{4}$ Department of Cardiology, San Martino University-Hospital, Genova, Italy \\ ${ }^{5}$ Presidio Ospedaliero F. Ferrari Casarano, Lecce, Italy \\ ${ }^{6}$ Kardiologische Gemeinschaftspraxis Kaltofen Schubert Gerner, Chemnitz, Germany \\ ${ }^{7}$ Ospedale Fabrizio Spaziani, Frosinone, Italy \\ ${ }^{8}$ Middle Slovak Institute of Cardiovascular Diseases, Banská Bystrica, Slovakia \\ ${ }^{9}$ Servicio de Cardiología, Hospital Universitario NuestraSeñora de Candelaria, Santa Cruz de Tenerife, Spain \\ ${ }^{10}$ Ospedale Civile San Leonardo, Castellammare di Stabia, Italy \\ ${ }^{11}$ Boston Scientific, St. Paul, MA \\ ${ }^{12}$ National Institute of Heart Diseases NUSCH, Bratislava, Slovakia
}

\begin{abstract}
The aim was to observe the relationship of pacemaker programming and patients' outcome in an unselected population of recipients in real-world practice. Pacemaker programming at hospital discharge and at scheduled follow-up visits was recorded and classified as physiologic based on simple principles: avoidance of unnecessary ventricular stimulation in patients without atrioventricular block, avoidance of unnecessary atrial stimulation in those without sinus node dysfunction, rate increase in those with chronotropic incompetence, and avoidance of hypotension-related symptoms. All-cause 2-year mortality was the primary endpoint; atrial fibrillation (AF) episodes leading to unplanned visits/hospitalizations was the secondary endpoint. A total of 1740 patients were followed for $24 \pm 3$ months, $89 \%$ had $D D D(R)$ pacemakers; physiologic programming was not associated with better survival, but it was associated with a lower incidence of $A F$ episodes in the 810 patients with $A V$ block as primary pacemaker indication (6.5\% versus $11 \%, p<0.01)$. Physiologic pacemaker programming occurred in only $41.4 \%$ of patients at hospital discharge; reprogramming towards a physiologic setting occurred in $8.7 \%$ at follow-up, mainly (76\%) within the first 6 months. Physiologic programming is associated with a lower incidence of $A F$ episodes in $A V$ block patients beyond maintenance of $A V$ synchrony. Proactive pacemaker behavior to increase physiologic programming should be considered, in view of physicians' reluctance to change programming from shipment settings.
\end{abstract}

KEYWORDS. Atrial fibrillation, device automaticity, physiologic programming.
ISSN 2156-3977 (print) ISSN 2156-3993 (online)

(C) 2016 Innovations in Cardiac Rhythm Management

\footnotetext{
Mauro Biffi and Giuseppe Boriani have a financial relationship with one or more companies that produce or market products or services relevant to the topic of this manuscript. Specifically, the authors disclose the following financial relationship: Mauro Biffi reported educational activity and speaker's bureau affiliated with Biotronik, Boston Scientific, and Medtronic. Giuseppe Boriani reported speaker's fees affiliated with Boston Scientific, Medtronic, St. Jude, and Boehringer.

Manuscript received November 28, 2015, final version accepted December 15, 2015.

Address correspondence to: Mauro Biffi, MD, Institute of Cardiology, University of Bologna, Policlinico S. Orsola-Malpighi, Via Massarenti n.9, 40138 Bologna, Italy. E-mail: mauro.biffi@aosp.bo.it
} 


\section{Introduction}

Over the past decades, cardiac pacing has become a wellestablished treatment for symptomatic bradyarrhythmias. Because of advances in technology, the goals of permanent pacing are no longer confined to the prevention of syncope, but are also focused on improving quality of life and decreasing cardiovascular morbidity. Over the past decades several landmark trials have compared a number of cardiovascular outcomes among patients randomized to different pacing modalities. ${ }^{1-5}$

However, only recently a general consensus on the optimal recommended pacing mode for selected rhythm disorders has been agreed and incorporated into a statement on pacemaker (PM) mode selection from HRS/ACCF. ${ }^{6}$ Dualchamber pacing or single-chamber atrial pacing is recommended over single-chamber ventricular pacing in patients with sinus node dysfunction, and for patients with atrioventricular block (AVB) except in specific clinical situations (e.g. sedentary patients or those with multiple comorbidities). Beyond pacing mode selection, several specific programmable options are available in modern PMs, in favor of pacing mechanisms that could mimic the physiologic function of the heart. These include an optimized management of the programmed pacing rate (lower rate, rate hysteresis, rate responsiveness), of atrioventricular $(\mathrm{AV})$ conduction (automatic search of intrinsic $\mathrm{AV}$ conduction or reversion from AAI to DDD mode), and other specific algorithms for reduction of symptoms. However, benefit from "physiologic pacing" has not been conclusively demonstrated in any of the landmark clinical trials. The OPTI-MIND (Clinical Outcome of Pacemaker paTlents according to pacing Modality and primary INDications) study was designed to collect data on midterm clinical outcome for PM patients and to stratify both clinical characteristics and outcome of these subjects based on the "real-world" choice of devices and their settings when analyzed with respect to a theoretically "physiologic programming."

\section{Methods}

\section{Study design}

OPTI-MIND is an observational, prospective, multicenter, single-arm cohort study (ClinicalTrials.gov Identifier: NCT00976482). The OPTI-MIND study is aimed at collecting data on device programming management and mid-term clinical outcomes according to real-world clinical practice in a group of patients implanted with a permanent PM (ALTRUA, Boston Scientific Corporation, Marlborough, MA) according to current practice. Patients were followed for a period of 2 years after enrolment according to the schedule and standard practice at the enrolling centers after signing an informed consent as per the local EC recommendations. The primary endpoint of the study was to document the 2-year all-cause mortality, in the whole cohort and stratify per patient characteristics, and per the chosen pacing modality against a specific set of device programming (physiologic pacing setting) on the basis of the primary indications for PM implant.
A pre-specified set of device programming options was identified in order to classify pacing modality as "physiologic" or "non-physiologic" in all subjects based on the following guiding principles: maintenance of AV synchrony, avoidance of unnecessary right ventricular (RV) stimulation in patients without AVB, avoidance of unnecessary atrial stimulation in patients without sinus node dysfunction (SND), rate increase during exercise, and avoidance of hypotension-related symptoms. These conditions were translated into defined PM programming options, each one specific to a patient's primary rhythm disease (i.e. primary reason for implant), concomitant rhythm disturbances, and the presence of concomitant chronotropic disease, as summarized in Table 1. These criteria were used as a surrogate definition of physiologic pacing and used together with clinical variables to stratify clinical events of primary endpoint. According to the pre-specified secondary study endpoints ${ }^{7}$ we investigated 1) the difference in the composite of cardiac cause mortality or first cardiac-related hospitalization between groups programmed with physiologic versus non-physiologic settings at baseline; 2) incidence at 2 years of documented atrial fibrillation (AF) episodes associated with any unplanned hospital visit, including hospitalizations for symptoms and/or treatment of AF in the subgroup of patients without $\mathrm{AF}$ as the primary rhythm disease at enrolment; 3) comparison between pacing settings at discharge and final programmed pacing parameters at 2 years; 4 ) clinical development of indications and/or upgrade to implantable cardioverter-defibrillator (ICD) or cardiac resynchronization device (CRT). Details on study design, together with analysis of baseline data have been previously published. ${ }^{7}$ Baseline device programming settings obtained at a subject's enrollment, occurring per study protocol in between 1 and 15 days since device implant were used to define patients programmed to physiologic pacing setting or not. Modifications in device programming during follow-up leading to a potential change of group were analyzed by comparing final programming setting (at 2 years or latest available) to the original one. Analysis of all study endpoints considered data collected up to 2 years after enrollment. In order to reliably capture patient status at the study end, without forcing a specific visit due to the observational design, a follow-up window of $24 \pm 3$ months was considered acceptable for documenting the patient vital status at study end.

\section{Statistical analysis}

The primary endpoint was calculated by using a KaplanMeier estimate at an alpha-level of 0.05. There was no formal statistical hypothesis for the primary endpoint: an $11 \%$ all-cause mortality incidence at 2 years was assumed per protocol. The aim was to achieve a precision of $3.2 \%$ (a distance from the proportion limit: 1.6\%) for the estimate of all-cause mortality in the entire group of enrolled patients. Death was considered as a study primary event and device upgrade was considered as censored data at the time of the occurrence. The log-rank test was used to evaluate differences in all-cause mortality and the composite of first cardiac-cause hospitalization or mortality 
with respect to physiologic and non-physiologic programming at baseline. Cox proportional hazards model were used to analyze multivariate association of all-cause mortality and new-onset atrial tachyarrythmias (in the subset of patients with AV block), with physiologic pacing setting (Table 1), adjusted for a set of pre-defined variables: gender, age $>80$ years, primary indication for pacing (SND, AVB, permanent AF with slow ventricular response, neuro-mediated syndrome), left ventricular ejection fraction (LVEF), underlying cardiomyopathy, number of associated diseases ( $>1$ of hypertension, diabetes, renal disease, chronic pulmonary disease, peripheral artery disease, other malignancies, or chronic diseases), resting heart rate at baseline, atrial based pacing mode. Variables associated with the clinical events with a $\mathrm{p}<0.1$ on univariate analysis were used in the model. The physiologic pacing group was classified according to device programming at enrolment, regardless of changes occurring during follow-up (intention to treat analysis). The lower bound of the confidence interval was compared to 1 . The sample size of the study was sufficient to detect a minimum difference of $8.50 \%$ versus $12.85 \%$ between the physiologic and the non-physiologic pacing setting, with a two-sided significance level (alpha) of 0.05 , an $80 \%$ power and an exponential drop-out rate of 0.075 (per year).

Descriptive statistics were used to summarize the observational data collected in this clinical study. For continuous variables the mean, standard deviation, and ranges may be used to represent the data. For discrete variables, frequency distributions may be presented. Parametric and non-parametric statistical tests were performed when appropriate. The analysis will be of an exploratory nature and performed continuously. Statistical analyses were conducted using SAS version 9.1 (SAS Institute, Cary, NC).

\section{Results}

\section{Enrollment and baseline data}

A total of 1740 subjects were enrolled in the study at 68 centers worldwide between September 2009 and June 2011. A summary of basic demographics, medical history, and clinical characteristics is reported in Table 2: 693/ $1674(41.4 \%)$ subjects received a physiological pacing setting at the baseline visit. ${ }^{7}$ Among the 981 patients not programmed with physiologic settings, causes of nonphysiological pacing included absence of AV synchrony through the appropriate pacing mode (183, 10.9\%); no avoidance of unnecessary right atrial pacing (with lower rate limit equal or above $60 \mathrm{ppm}$ ) in subjects without sinus node dysfunction (440,26.3\%); no avoidance of unnecessary RV stimulation in subjects without $\mathrm{AV}$ conduction block $(178,10.6 \%)$, and absence of a rate increase during exercise with a rate responsive function $(180,10.8 \%)$. As previously reported, ${ }^{7}$ there were no

Table 1: Physiologic pacing settings definition

\begin{tabular}{|c|c|c|c|}
\hline $\begin{array}{l}\text { Primary rhythm } \\
\text { indication }\end{array}$ & $\begin{array}{l}\text { Additional rhythm } \\
\text { disease }\end{array}$ & $\begin{array}{l}\text { Chronotropic } \\
\text { incompetence }\end{array}$ & Physiologic settings (logic) \\
\hline \multirow[t]{4}{*}{ AVB } & Without SND & Yes & $\begin{array}{l}\text { ((DDDR) AND }(\mathrm{LRL}<60 \text { bpm or rate hysteresis }=\mathrm{ON})) \text { or }((\mathrm{VDDR}) \\
\text { AND }(\mathrm{LRL}<60 \mathrm{bpm} \text { or rate hysteresis }=\mathrm{ON}))\end{array}$ \\
\hline & & No & $\begin{array}{l}\text { ((DDD OR DDDR) AND }(\mathrm{LRL}<60 \mathrm{bpm} \text { or rate hysteresis }=\mathrm{ON})) \text { or } \\
((\mathrm{VDD} \text { OR VDDR) AND }(\mathrm{LRL}<60 \mathrm{bpm} \text { or rate hysteresis }=\mathrm{ON}))\end{array}$ \\
\hline & With SND & Yes & DDDR \\
\hline & & No & DDD OR DDDR \\
\hline \multirow[t]{4}{*}{ SND } & Without AVB & Yes & $\begin{array}{l}\text { (AAIR) or (DDDR or DDIR) AND }((\text { AV search }=\text { ON }) \text { OR } \\
\left.\left(\text { AV } \min >P^{*}\right)\right)\end{array}$ \\
\hline & & No & $\begin{array}{l}\text { (AAI OR AAIR) or (DDD OR DDDR or DDI or DDIR) AND } \\
\left(\left(A V \text { search }=\text { ON) OR }\left(A \vee \min >P^{*}\right)\right)\right.\end{array}$ \\
\hline & With AVB & Yes & (DDDR) \\
\hline & & No & (DDD OR DDDR) \\
\hline \multirow[t]{8}{*}{ Neuromediated } & $\begin{array}{l}\text { Without AVB or } \\
\text { SND }\end{array}$ & Yes & $\left(\mathrm{DDIR}\right.$ or DDDR) AND ((rate hysteresis $\left.=\mathrm{ON}^{*}\right)$ or $\left.\mathrm{LRL}<60 \mathrm{bpm}\right)$ \\
\hline & & No & $\begin{array}{l}\text { (DDI OR DDIR) AND }\left(\left(\text { rate hysteresis }=\mathrm{ON}^{*}\right) \text { or } \mathrm{LRL}<60 \mathrm{bpm}\right) \text { or } \\
\left(\left(\mathrm{DDD} \text { or DDDI) AND }\left(\left(\text { rate hysteresis }=\mathrm{ON}^{*}\right) \text { or } \mathrm{LRL}<60 \mathrm{bpm}\right)\right.\right. \\
\text { AND }\left((\mathrm{AV} \text { search }=\mathrm{ON}) \text { OR }\left(A V \text { min }>\mathrm{PR}^{*}\right)\right)\end{array}$ \\
\hline & AVB only & Yes & $\begin{array}{l}((\mathrm{DDDR}) \text { AND }(\mathrm{LRL}<60 \text { bpm or rate hysteresis }=\mathrm{ON})) \text { or }((\mathrm{VDDR}) \\
\text { AND }(\mathrm{LRL}<60 \mathrm{bpm} \text { or rate hysteresis }=\mathrm{ON}))\end{array}$ \\
\hline & & No & $\begin{array}{l}\text { ((DDD OR DDDR) AND }(\mathrm{LRL}<60 \mathrm{bpm} \text { or rate hysteresis }=\mathrm{ON})) \text { or } \\
((\text { VDD OR VDDR) AND }(\mathrm{LRL}<60 \mathrm{bpm} \text { or rate hysteresis }=\mathrm{ON}))\end{array}$ \\
\hline & SND only & Yes & $($ AAIR $)$ or (DDDR) AND $\left((\right.$ AV search $=$ ON $)$ OR $\left.\left(A \vee \min >P^{*}\right)\right)$ \\
\hline & & No & $\begin{array}{l}\text { (AAI OR AAIR) or (DDD OR DDDR) AND }((A \vee \text { search }=\text { ON }) \text { OR } \\
\left.\left(A \vee \min >P^{*}\right)\right)\end{array}$ \\
\hline & $\begin{array}{l}\text { Both AVB and } \\
\text { SND }\end{array}$ & Yes & (DDDR) \\
\hline & & No & (DDD OR DDDR) \\
\hline Permanent AF & Any & Any & $\begin{array}{l}\text { (DDD or DDDR or DDI or DDIR or VDD or VVIR) OR (VVI AND } \\
\text { algorithms for } A F=\mathrm{ON}^{*} \text { ) }\end{array}$ \\
\hline
\end{tabular}

AF: atrial fibrillation; AVB: atrioventricular block; SND: sinus node dysfunction. 
differences in the medical history and in the most important clinical characteristics (type of underlying disease, cardiovascular risk factors, LVEF, heart failure status, comorbidities) of patients receiving a physiologic programming with respect to a non-physiologic one. The use of antiarrhythmic drugs was slightly higher in patients with physiologic programming, with a significant difference between primary indications for pacing $(\mathrm{p}<0.01)$ : it was $18 \%(96 / 538)$ in SSS patients $(52 / 251$ patients with physiological setting versus $44 / 287$ patients with non-physiological setting, $\mathrm{p}=0.10) ; 4.6 \%(37 / 810)$ in AVB patients (11/260 patients with physiological setting versus $26 / 550$ with non-physiological setting, $\mathrm{p}=0.75$ ); $8 \%(5 / 62)$ in neuro-mediated patients (3/34 patients with physiological setting versus $2 / 28$ with non-physiological setting, $\mathrm{p}=0.81)$; and $5 \%(12 / 264)$ in permanent $A F$ patients $(8 / 148$ patients with physiological setting versus $4 / 116$ with non-physiological setting, $\mathrm{p}=0.45$ ).

\section{Follow-up and clinical outcome}

During the course of the study, from enrollment until last follow-up (24 \pm 3 months) and up to 831 days of enrolment period, $208(11.9 \%)$ patients died, and $45(2.6 \%)$ withdrew from the study. Withdrawals included device explant because of upgrade to ICD or CRT $(24,1.3 \%)$, patient voluntary withdrawal $(11,0.6 \%)$, patient loss to follow-up $(10,0.5 \%)$.

Among the 208 deaths recorded in the database, 194 $(11.1 \%)$ occurred within a 24-month window after subject enrollment and were used for primary endpoint analysis. This resulted in a total $11 \%$ incidence at 2 years, consistent with the original protocol assumption. Cause of death was identified as cardiac in 51 cases $(2.9 \%)$, noncardiac in $86(4.9 \%)$, and unknown in the remaining 71 patients $(4 \%)$. None of the deaths was related to implanted device malfunctions. No unanticipated adverse device effects or serious adverse device effects were identified in the present study.

All-cause mortality along the 2 years of follow-up is reported in Figure 1. The multivariate Cox proportional hazards model for 2-year all-cause mortality identified older age and compromised ejection fraction as independent predictors of mortality (Table 3). Physiologic pacing programming at baseline was not associated with lower mortality (adjusted HR, 0.97; 95\% CI, 0.66-1.42; $\mathrm{p}=0.97$ ). A total of 567 subjects (32.6\%) experienced at least one hospitalization during the course of the study. No differences in the composite of first cardiac-cause hospitalization or mortality were detected when comparing patients with device programmed versus not programmed with physiologic pacing settings (11.2\% versus $10.7 \% ; \mathrm{p}=0.74)$.

\section{New-onset atrial fibrillation}

Among 1410 subjects without AF as primary rhythm disease at enrollment (538 with sinus node dysfunction, 810 with AV block, and 62 with neurally mediated syndrome), 206 (14.6\%) experienced at least one episode of AF or tachycardia during 2 years of follow-up: $13.1 \%$ in the physiologic pacing group versus $15.8 \%$ in non-physiologic pacing group $(\mathrm{p}=0.21)$. When considering subgroups based on primary rhythm disease, $\mathrm{AF}$ incidence was $21 \%(113 / 538)$ in SSS patients $(48 / 251$ with physiological setting versus $65 / 287$ with nonphysiological setting, $\mathrm{p}=0.21) ; 10.2 \%(83 / 810)$ in AVB patients (17/260 patients with physiological setting versus $66 / 550$ with non-physiological setting, $\mathrm{p}=0.01$ ); $16 \%(10 / 62)$ in neuro-mediated patients (2/34 patients with physiological setting versus $8 / 28$ with non-physiological setting, $\mathrm{p}=0.02$ ). Figure 2 displays the 2-year incidence of new-onset atrial tachyarrhythmias in this numerically consistent subgroup. In 527 subjects with AV block, the major causes for non-physiologic programming were absence of AV synchrony because of pacing mode (82 patients; VVI, 44; VVIR, 29; DDIR, 6; DDI, 3) and unnecessary atrial stimulation in the absence of SND $(435 / 527,82 \%)$. A multivariate Cox proportional hazards model applied to this subgroup of patients with AV block (Table 4) identified non-physiological pacing setting and increased baseline heart rate at electrocardiography as strong independent predictors of the development of $\mathrm{AF}$ or tachycardia during the 2 years follow-up period, with both $\mathrm{D}(\mathrm{R})$ pacing mode (adjusted HR, $0.2 ; 0.12-0.32 ; \mathrm{p}<0.001)$ and lowering the lower rate limit (LRL) below 60 ppm (adjusted HR, 0.59; 0.35-0.99; $\mathrm{p}=0.046$ ) being associated with significant relative reduction of atrial arrhythmias.

\section{Changes in device programming}

Enrolled subjects underwent a median of three ambulatory visits (interquartile range, 2-4) during the 2 years of follow-up in the study, with $55 \%$ of them having the first visit within 3 months from enrollment, 21\% between 3 and 6 months and 24\% after 6 months. During ambulatory visits, a programming change of one or more of the studyrelevant pacing parameters occurred in 335 patients $(19.2 \%)$. Changes included pacing mode modification (except rate response changes) in 76 patients $(4.3 \%)$, lower rate limit change (above or below the $60 \mathrm{ppm}$ rate) in 103 $(5.9 \%)$, minimum/maximum $\mathrm{AV}$ delay or $\mathrm{AV}$ interval search function modification in 199 (11.4\%), rate hysteresis activation/deactivation in $80(4.6 \%)$, rate response turned on/off in $217(12.5 \%)$, and rate response optimization in $42(2.4 \%)$. Among these changes, the modified settings resulted in an optimization toward physiologic pacing in $147 / 335$ patients only $(44 \%)$, the majority of the reprogramming (112/147, 76\%) occurring within 6 months from enrolment. The factors determining the switch to physiologic pacing are presented in Figure 3, the main being the activation of rate response in patients with permanent $\mathrm{AF}$ at baseline.

\section{Discussion}

This study reports on PM programming in the current practice of cardiac stimulation over an unselected cohort of patients. We observed that PM programming is driven neither by the pathophysiology of rhythm disease nor by recently published recommendations. ${ }^{6}$ Indeed, only $46.6 \%$ of patients with SSS and 28\% of AVB patients in sinus 
Table 2: Baseline characteristics

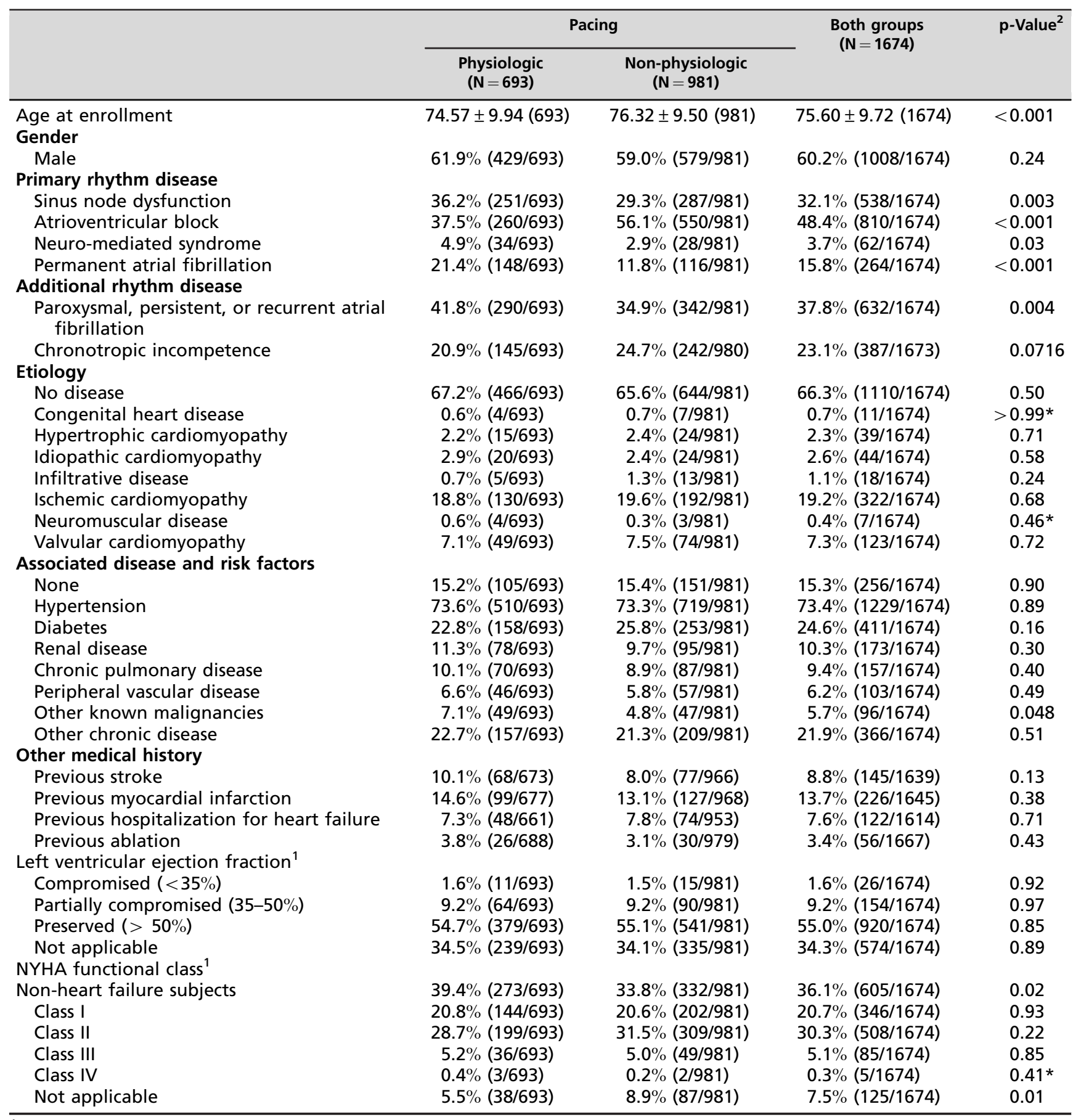

${ }^{1}$ Last measurement within 12 months.

${ }^{2} \mathrm{p}$-Value from the chi-square test unless noted with an asterisk, wherein the Fisher exact test was used.

rhythm received a strictly physiologic programming according to the generic principles of maintenance of AV synchrony, avoidance of unnecessary atrial/ventricular pacing when not indicated, allowance of rate increase in chronotropically incompetent patients. ${ }^{7}$ Physiologic programming was not associated with increased mortality or hospitalizations. However, in the subgroup with AVB as primary rhythm disease, physiologic pacing was associated with a lower incidence of new-onset atrial tachyarrhythmias. As the primary study endpoint was not met, the results about secondary endpoints should be interpreted cautiously and as hypothesis-generating, given the exploratory and nonrandomized nature of the design.

\section{Mortality and predictors}

At multivariate analysis all-cause mortality was associated with older age and LV systolic dysfunction and not 


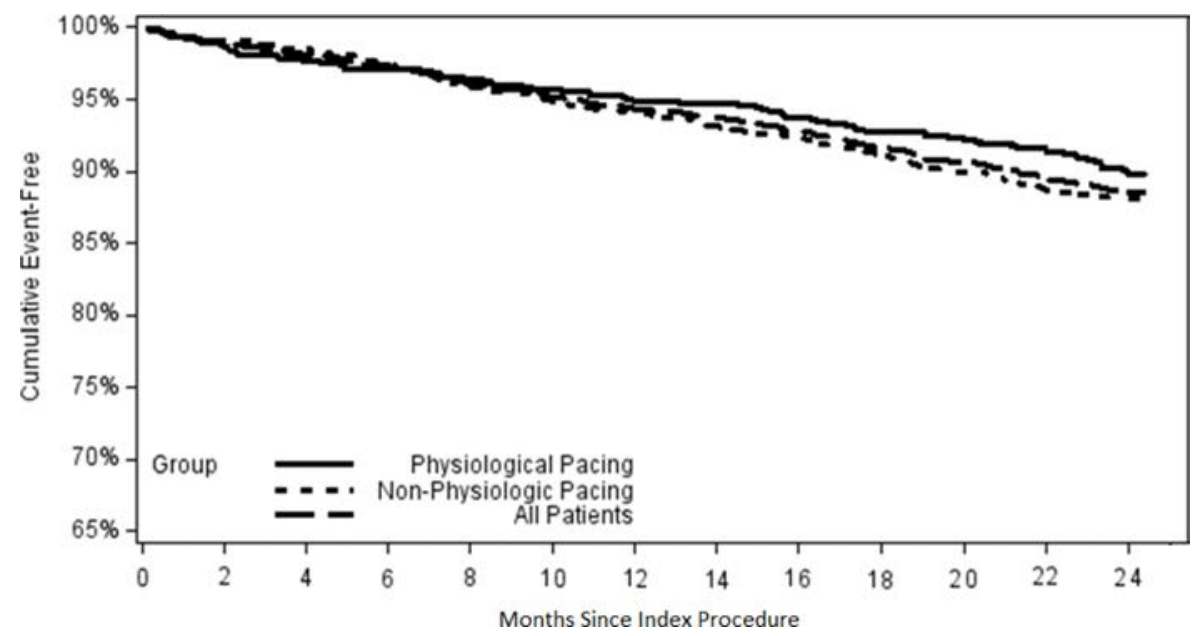

Figure 1: Two-year all-cause mortality cumulative incidence according to physiologic pacing settings.

with physiologic programming. Physiologic programming occurred most frequently in patients with SND and with AF as primary rhythm disease, whereas non-physiologic pacing was more frequently observed in patients with $\mathrm{AVB},{ }^{7}$ showing that evidence-based indications have a partial impact on pacing mode selection and device programming for SND patients, while non-physiologic pacing is more common in AVB patients. Indeed, AV synchrony was not maintained in $10 \%$ of AVB patients and unnecessary atrial stimulation was delivered in $59 \%{ }^{7}$ Although loss of $\mathrm{AV}$ synchrony was unevenly distributed across the primary rhythm indications for pacing, it accounted overall for only $11 \%$ of patients. ${ }^{7}$ As loss of AV synchrony is reported to be the major determinant of cardiovascular and total mortality in patients without permanent $\mathrm{AF}^{8}$ the limited proportion of these cases found in the study may reasonably explain the lack of association between physiologic programming and all-cause mortality. Also in our population, no association was found between the intrinsic rate recorded before PM implantation ( $>$ or $<50 \mathrm{bpm}$ ) and mortality (Table 3), possibly due to the high level of maintenance of AV synchrony and of avoidance of unnecessary RV stimulation observed across the whole patient population. ${ }^{7}$ This is in agreement with the observations stemming from both the CTOPP and the MOST sub-studies. ${ }^{8,9}$

Table 3: Multivariate Cox proportional hazards model for 2-year all-cause mortality

\begin{tabular}{lcr}
\hline Putative predictors & \multicolumn{1}{c}{$\begin{array}{c}\text { Adjusted hazard } \\
\text { ratio }(95 \% \mathrm{Cl})\end{array}$} & $\mathrm{p}$-Value \\
\hline Physiological pacing & $0.97(0.66$ to 1.42$)$ & 0.8674 \\
Age $>80$ years & $2.78(1.90$ to 4.06$)$ & $<0.0001$ \\
Sinus node dysfunction & $0.93(0.61$ to 1.42$)$ & 0.7230 \\
$\begin{array}{c}\text { Atrial based pacing mode: } \\
\quad \text { dual chamber }\end{array}$ & $0.75(0.50$ to 1.11$)$ & 0.1472 \\
$\begin{array}{l}\text { Intrinsic heart rate }>50 \\
\text { Left ventricular ejection } \\
\quad \text { fraction }>50 \%\end{array}$ & $1.18(0.79$ to 1.76$)$ & 0.4303 \\
$\begin{array}{c}\text { Number of associated } \\
\quad \text { diseases }>1\end{array}$ & $1.34(0.92$ to 0.67$)$ & $<0.0001$ \\
\hline
\end{tabular}

\section{Device programming and $A F$}

Our definition of physiologic pacing also comprised avoidance of unnecessary atrial/ventricular stimulation and allowance of rate increase in the event of chronotropic incompetence according to the patient's profile. We observed that physician behavior at reprogramming from shipment parameters towards a strictly physiologic setting is different in AVB patients with respect to other rhythm diseases. ${ }^{7}$ Beyond lesser maintenance of AV synchrony, unnecessary atrial stimulation occurred in $59 \%$ of AVB patients because of an unchanged lower rate. Both aspects were strongly associated with an increased AF incidence (Table 4), with a low AF occurrence early in the follow-up period in subjects programmed with physiologic pacing (Figure 2). It is known that $\mathrm{AV}$ synchrony and avoidance of unnecessary atrial stimulation ensure optimal diastolic filling of the left ventricle ${ }^{10-12}$ and prevent atrial dyssynchrony, ${ }^{13-15}$ thereby decreasing AF incidence. Optimization of diastolic filling time is a key factor to ensure clinical response to $\mathrm{CRT}^{11}$ but is most frequently disregarded in AVB patients. Indeed, the occurrence of atrial stimulation at the lower rate may create interatrial dyssynchrony with a "functionally short" AV delay and a truncated or absent A wave $\mathrm{e}^{12}$ as in Figure 4, resulting in increased left atrial pressure.

The extent of suboptimal hemodynamics related to an untailored paced AV delay is presently unknown in medical literature, and may also have played a role in randomized studies which failed to demonstrate a mortality benefit of dual-chamber versus single-chamber stimulation. ${ }^{1,2}$

The achievement of an appropriate diastolic filling by AV delay optimization has emerged also in SSS, DDDR pacing having a lower incidence of AF than AAIR pacing in the DANPACE study. ${ }^{16}$ Regretfully, in AVB patients the pacing rate is not lowered to allow VDD pacing and paced AV delay is not targeted to ensure the best diastolic filling. Indeed, the paced AV delay and the shipment offset between paced and sensed AV delay differ among manufacturers, may show individual differences among 


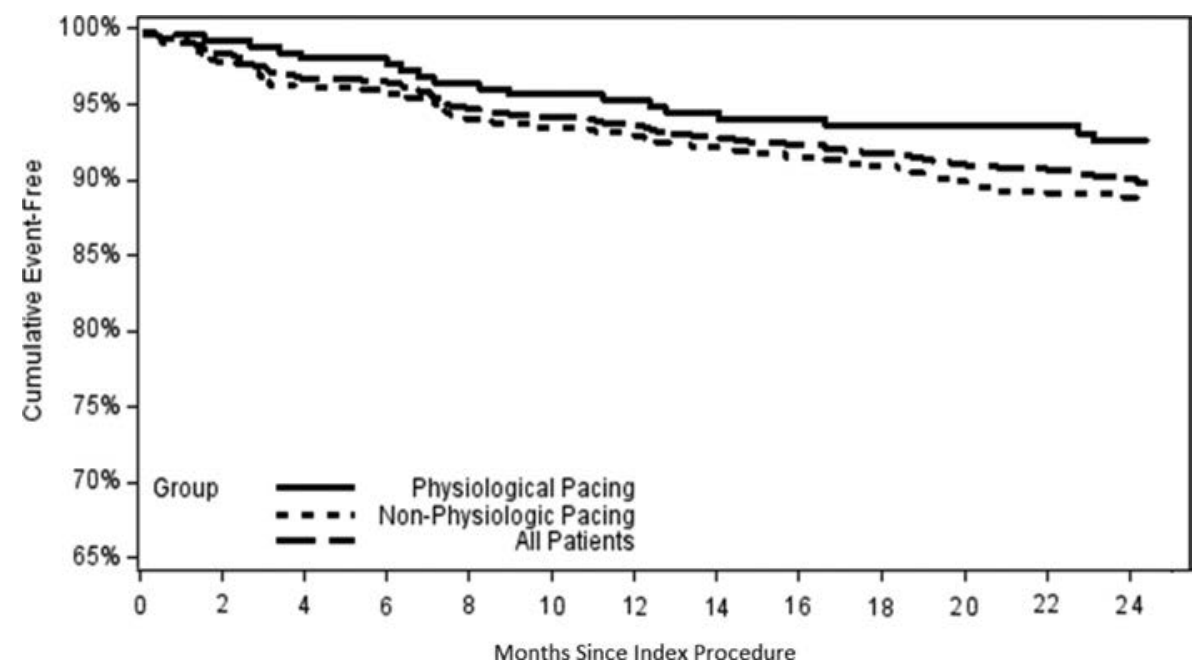

Figure 2: Two-year incidence of atrial fibrillation or tachycardia in the subgroup of subjects with atrioventricular block as primary rhythm disease according to physiologic pacing settings.

patients, are strongly dependent on interatrial conduction that is only sporadically considered, and are only rarely changed at follow-up. As expected, an intrinsic rate $>50 \mathrm{bpm}$ before PM implantation was linked with higher incidence of new-onset atrial arrhythmias, since avoidance of unnecessary RV pacing and optimization of diastolic filling at the lower rate by $\mathrm{AV}$ delay programming may not have occurred, as in agreement with the MOST and CTOPP ${ }^{8,9}$ observations and CRT lessons. ${ }^{10-12}$ Indeed, only $11.4 \%$ patients had AV delay reprogramming towards a more physiologic setting during follow-up.

\section{Pacemaker reprogramming during follow-up}

An additional exploration of these data investigated how device programming changes over time, according to current clinical practice. Patients in this study were followed with a median of three in-clinic follow ups over a 2-year timeframe, with first visit occurring in approximately half of the subjects within 3 months from enrollment. However, when looking at programming changes and device setting

Table 4: Multivariate Cox proportional hazards model for 2-year events of atrial fibrillation or tachycardia in the subgroup of subjects with atrioventricular block as primary rhythm disease

\begin{tabular}{lcc}
\hline Effect & $\begin{array}{c}\text { Adjusted hazard } \\
\text { ratio }(95 \% \mathrm{Cl})\end{array}$ & p-Value \\
\hline $\begin{array}{c}\text { Physiological pacing }-\mathrm{D}(\mathrm{R}) \\
\text { pacing mode versus } \mathrm{V}(\mathrm{R})\end{array}$ & $0.2(0.12$ to 0.32$)$ & $<0.001$ \\
$\begin{array}{c}\text { Physiological pacing - pacing } \\
\text { rate }<60 \text { ppm }\end{array}$ & $0.59(0.35$ to 0.99$)$ & 0.046 \\
$\begin{array}{l}\text { Age }>80 \text { years } \\
\text { Intrinsic heart rate }>50\end{array}$ & $0.92(0.57$ to 1.49$)$ & 0.758 \\
$\begin{array}{l}\text { Left ventricular ejection } \\
\text { fraction }>50 \%\end{array}$ & $0.73(0.47$ to 1.14$)$ & 0.173 \\
$\begin{array}{l}\text { Number of associated } \\
\text { diseases }>1\end{array}$ & $1.34(0.86$ to 2.08$)$ & 0.190 \\
\hline
\end{tabular}

during follow-up, only 147 (8.4\%) of the patients underwent a programming change toward physiological programming. Furthermore, when analyzing specific device setting changes in these patients, the principal adjustment was activation of rate response in patients with permanent AF $(49 / 147 ; 33 \%)$, while only the minority of the subjects underwent a change to achieve a substantial enhancement of physiologic pacing: in patients with AVB and without SND the paced AV was reprogrammed in 19/147 (13\%), and the LRL was lowered below $60 \mathrm{ppm}$ in 24/147 (16\%). Additionally, most of these changes (76\%) occurred within 6 months of enrollment. This picture shows that little attention is paid to device optimization, the majority of reprogramming occurring at the time of implant, or at the earliest follow-up visit. Consistently with this finding, in the AUTOMATICITY trial ${ }^{17} \mathrm{PM}$ algorithm reprogramming occurred rarely, and was not driven by a patient's clinical needs in one-third of cases.

\section{Study limitations}

The relevance of our study is limited by the nonrandomized design of the study and absence of hypothesis testing. However, the value of the Registry stems from the strong adherence to the real clinical practice of cardiac stimulation in a broad unselected population across different countries.

\section{Conclusions}

The OPTI-MIND study showed that in current clinical practice, a "physiologic," as per this study definition, programming of PM at baseline visit did not increase overall survival, AV synchrony being maintained in $89 \%$ of patients with sinus rhythm. On the contrary, the physiologic PM setting was associated with a lower AF incidence in AVB patients. The behavior of physicians shows reluctance at device re-programming towards a more physiologic setting during follow-up of patients, the main parameters changes occurring within the first 


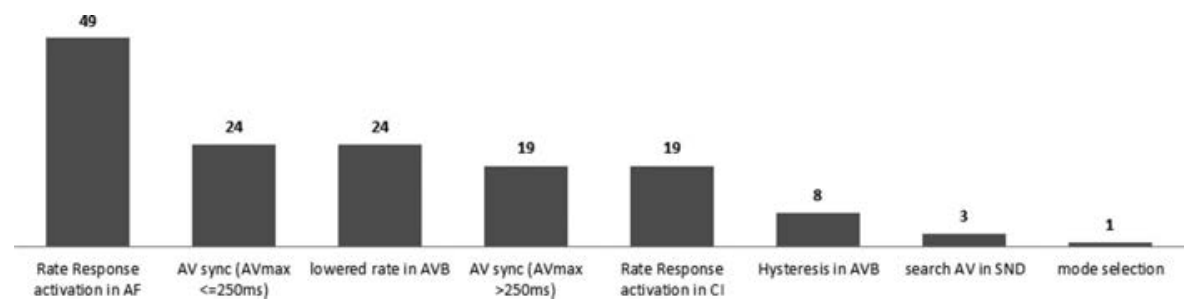

Figure 3: Drivers of pacemaker re-programming to a physiologic setting. AF: Atrial Fibrillation; AV atrio-ventricular; AVB: atrio-ventricular block; $\mathrm{Cl}$ : chronotropic incompetence.
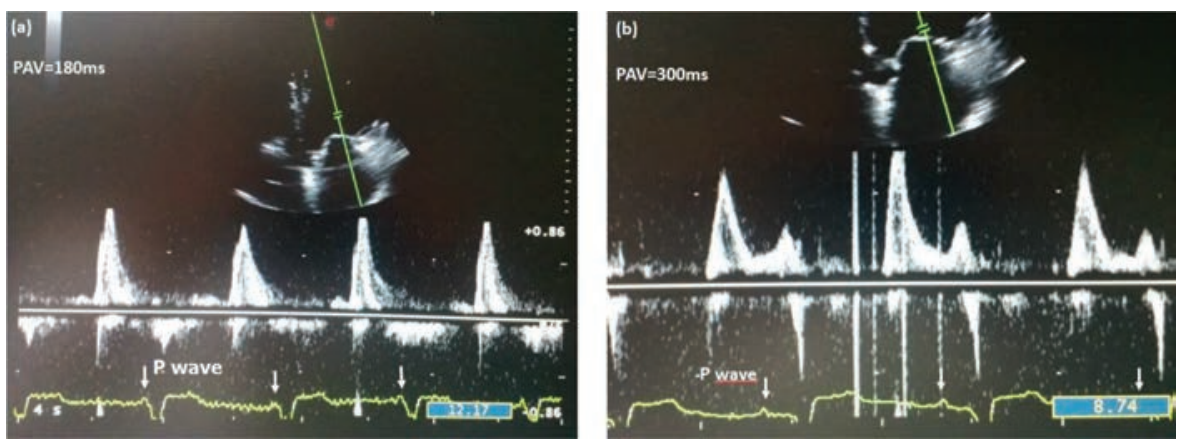

Figure 4: Echographic evidence of interatrial dyssynchrony with a "functionally short" atrioventricular delay (a) and optimization of diastolic filling time (b).

6 months, and being mainly focused on rate-response activation in AF patients. This sets the basis for proactive behavior on the device side in future PM releases, aiming to ensure tailored AV synchrony, unnecessary atrial/ ventricular stimulation avoidance, and automatic increase to an individually targeted heart rate when patient activity is detected and unmatched by the patient's intrinsic rhythm, within ranges individually defined by the clinician.

\section{Appendix: List of participating sites}

List of Principal Investigators, participating sites and enrolled subjects in alphabetical order per country and city: Frank Heinzel, Medizinische Univ.-Kliniken Graz, Graz, Austria (23); Stefan Zorn, A.O. Krankenhaus der barmherzigen Schwestern Ried, Ried im Innkreis, Austria (18); Cesar Khazen, Allgemeines Krankenhaus AKH, Vienna, Austria (1); Petr Polasek, Kelowna General Hospital, Kelowna, Canada (23); Bernard Thibault, Institut de Cardiologie de Montreal, Montreal, Canada (20); Gilles O'Hara, Institut universitaire de Cardiologie et de Pneumologie de Quebec, Ste-Foy, Canada (53); Ales Havlicek, Pardubice Artur Koblitz, Pardubice, Czech Republic (29); Soren Hojberg, Bispebjerg Hospital, Copenhagen, Denmark (6); Thomas Melchior, Roskilde University Hospital, Roskilde, Denmark (24); Birger Engby, Vejle Sygehus, Vejle, Denmark (33); Per Christensen, Viborg Sygehus, Viborg, Denmark (9); Daniel Galley, Centre Hospitalier d'Albi, Albi, France (14); Zafer Alagha, Centre Hospitalier Jacques Coeur, Bourges, France (15); Eric Espaliat, Clinique Les Cèdres, Brive-La Gaillarde, France (15); Henri Dorey, CHP St. Martin, Caen, France (29); Yves Guyomar, Hôpital
Saint Philibert, Lomme, France (11); Olivier Bizeau, CHR d'Orléans-Hopital de la Source, Orléans, France (34); Gilles Lascault, Centre Cardiologique du Nord, St. Denis, France (25); Horst Neubauer, St. Josef Hospital, Bochum, Germany (14); Rüdiger Dissmann, Klinikum Reinkenheide Bremerhaven, Bremerhaven, Germany (39); Gert Kaltofen, Kardiologische Gemeinschaftspraxis , Chemnitz, Germany (70); Johann Mermi, Evangelisches Krankenhaus Giessen, Giessen, Germany (3); Gisbert Gehling, Johannes Hospital, Hagen, Germany (33); Ali Aydin, Universitätsklinik Eppendorf, Hamburg, Germany (16); Bernhard Zrenner, Krankenhaus Landshut Achdorf, Landshut, Germany (25); Christian Kuehne, Universitätsklinikum Leipzig, Leipzig, Germany (48); Ajay Naik, Care Institute of Medical Sciences, Ahmedabad, India (3); Ulhas Pandurangi, Madras Medical Mission, Chennai, India (3); Manjinder Singh Sandhu, Artemis Health Institute, Gurgaon, India (5); Anil Saxena, Escort Heart Institute and Research Centre, New Delhi, India (4); Giuseppe Boriani, Ospedale S. Orsola - Malpighi, Bologna, Italy (70); Donato Melissano, Pres. Osp. F. Ferrari, Casarano, Italy (70); Ciro Guastaferro, Ospedale Civile San Leonardo, Castellamare di Stabia, Italy (41); Donatella Gorini, Ospedale Bufalini - Marconi, Cesena, Italy (15); Maurizio Menichelli, Ospedale Fabrizio Spaziani, Frosinone, Italy (68); Paolo Rossi, Azienda Ospedaliera Universitaria Martino di Genova, Genova, Italy (70); Aldo Celentano, Ospedale dei Pellegrini, Napoli, Italy (11); Gianfranco Ciaramitaro, A.O. Universitaria Policlinico Paolo Giaccone, Palermo, Italy (20); Francesco Romeo, Azienda Ospedaliera Universitaria Policlinico Tor Vergata, Roma, Italy (30); Gemma Pelargonio, Policlinico Universitario Agostino Gemelli, Roma, Italy (70); Vittorio Giudici, Ospedale Bolognini, Seriate, Italy (44); Marco Brieda, Osp. S. Maria Degli Angeli, Pordenone, Italy (8); 
Davide Saporito, Ospedale Degli Infermi, Rimini, Italy (13); Michele De Benedictis, Ospedale Maggiore SS. Annunziata, Savigliano, Italy (12); Alexander Wardeh, Medisch Centrum Haaglanden $(\mathrm{MCH})$ - locatie Westeinde, Den Haag, Netherlands (19); Maurits J. Van der Veen, Ziekenhuis Gelderse Vallei, Ede, Netherlands (32); Peter Geerlings, St. Jans Gasthuis Weert, Weert, Netherlands (10); Victor Sanfins, Centro Hospitalar do Alto Ave, Guimaraes, Portugal (33); Jose Santos, Sao Francisco Xavier, Lisbon, Portugal (4); Gabriela Kaliska, SUSCCH a.s., Banska Bystrica, Slovakia (53); Tibor Malacky, National Institute of Cardiovascular Diseases NUSCH, Bratislava, Slovakia (41); José Luis Diago, Hospital General de Castellon, Castellon, Spain (11); Juan Benezet, Hospital General de Ciudad Real, Ciudad Real, Spain (7); Francisco Marrero, Consorcio de Tenerife, La Laguna, Spain (50); Juan Antonio Sieira Rodriguez, Hospital Lucus Augusti, Lugo, Spain (10); Javier Alzueta, Hospital Universitario Virgen De La Victoria, Malaga, Spain (14); Marcos Antonio Perez Veloso, Hospital de Orense, Orense, Spain (28); Josep Maria Alegret, Hospital de Sant Joan de Reus, Reus, Spain (18); Claudio Ledesma, Hospital Clinico Salamanca, Salamanca, Spain (42); Rafael Romero, Hospital Universitario Nuestra Senora de Candelaria, Santa Cruz de Tenerife, Spain (50); Sven Reek, Hirslanden Klinik Aarau, Aarau, Switzerland (12); Martin Fromer, Centre Hospitalier Universitaire Vaudois, Lausanne, Switzerland (11); Tiziano Moccetti, Cardiocentro Ticino, Lugano, Switzerland (17); Urs Eriksson, GZO Spital Wetzikon, Wetzikon, Switzerland (6); Grahame Keith Goode, Blackpool Victoria NHS Trust Direct, Blackpool, United Kingdom (15); Ian Beeton, St. Peters Hospital, Chertsey, United Kingdom (28); Ian Williams, Norfolk and Norwich University Hospitals NHS , Norwich, United Kingdom (41).

Acknowledgment. The authors would like to thank Elisa Vireca (Boston Scientific, Diegem, Belgium) for her contribution in designing and conducting the study.

\section{References}

1. Lamas GA, Orav EJ, Stambler BS, et al. Quality of life and clinical outcomes in elderly patients treated with ventricular pacing as compared with dual-chamber pacing. N Engl J Med. 1998;338(16):1097-1104.

2. Connolly SJ, Kerr CR, Gent M, et al. Effects of physiologic pacing versus ventricular pacing on the risk of stroke and death due to cardiovascular causes. Canadian Trial of Physiologic Pacing Investigators. N Engl J Med. 2000; 342(19):1385-1391.
3. Kerr CR, Connolly SJ, Abdollah H, et al. Canadian Trial of Physiological Pacing: Effects of physiological pacing during long-term follow-up. Circulation. 2004;109(3):357-362.

4. Lamas GA, Lee KL, Sweeney MO, et al. Ventricular pacing or dual-chamber pacing for sinus-node dysfunction. N Engl J Med. 2002;346(24):1854-1862.

5. Andersen HR, Nielsen JC, Thomsen PE, et al. Long-term follow-up of patients from a randomised trial of atrial versus ventricular pacing for sick-sinus syndrome. Lancet. 1997;350(9086):1210-1216.

6. Gillis AM, Russo AM, Ellenbogen KA, et al. HRS/ACCF expert consensus statement on pacemaker device and mode selection. J Am Coll Cardiol. 2012;60(7):682-703.

7. Biffi M, Melissano D, Rossi P, et al. The OPTI-MIND study: A prospective, observational study of pacemaker patients according to pacing modality and primary indications. Europace. 2014;16(5):689-697.

8. Tang AS, Roberts RS, Kerr C, et al. Relationship between pacemaker dependency and the effect of pacing mode on cardiovascular outcomes. Circulation. 2001;103(25):3081-3085.

9. Sweeney MO, Hellkamp AS, Ellenbogen KA, et al. Adverse effect of ventricular pacing on heart failure and atrial fibrillation among patients with normal baseline QRS duration in a clinical trial of pacemaker therapy for sinus node dysfunction. Circulation. 2003;107(23):2932-2937.

10. Whinnett ZI, Briscoe C, Davies JE, et al. The atrioventricular delay of cardiac resynchronization can be optimized hemodynamically during exercise and predicted from resting measurements. Heart Rhythm. 2008;5(3):378-386.

11. Verbrugge FH, Verhaert D, Grieten L, et al. Revisiting diastolic filling time as mechanistic insight for response to cardiac resynchronization therapy. Europace. 2013;15(12): 1747-1756.

12. Bertini M, Delgado V, Bax JJ, Van de Veire NRL. Why, how and when do we need to optimize the setting of cardiac resynchronization therapy? Europace. 2009;11(Suppl 5):v46-v57.

13. Xie JM, Fang F, Zhang Q, et al. Atrial dysfunction and interatrial dyssynchrony predict atrial high rate episodes: Insight into the distinct effects of right atrial appendage pacing. J Cardiovasc Electrophysiol. 2012;23(4):384-390.

14. Eicher JC, Laurent G, Mathé A, et al. Atrial dyssynchrony syndrome: An overlooked phenomenon and a potential cause of 'diastolic' heart failure. Eur J Heart Fail. 2012;14(3):248-258.

15. Laurent G, Eicher JC, Mathé A, et al. Permanent left atrial pacing therapy may improve symptoms in heart failure patients with preserved ejection fraction and atrial dyssynchrony: A pilot study prior to a national clinical research programme. Eur J Heart Fail. 2013;15(1):85-93.

16. Nielsen JC, Thomsen PE, Højberg S, et al. A comparison of single-lead atrial pacing with dual-chamber pacing in sick sinus syndrome. Eur Heart J. 2011;32(6):686-696.

17. Alings M, Vireca E, Bastian D, et al. Clinical use of automatic pacemaker algorithms: Results of the AUTOMATICITY registry. Europace. 2011;13(7):976-983. 